\title{
Dissecting the Syndrome of Schizophrenia: Progress toward Clinically Useful Biomarkers
}

\author{
Brian Dean ${ }^{1,2}$ \\ ${ }^{1}$ The Rebecca L. Cooper Research Laboratories, The Mental Health Research Institute, Locked bag 11, Parkville, VIC 3052, Australia \\ ${ }^{2}$ The Department of Psychiatry, The University of Melbourne, Parkville, VIC 3052, Australia
}

Correspondence should be addressed to Brian Dean, anddali@unimelb.edu.au

Received 18 December 2010; Revised 28 March 2011; Accepted 7 April 2011

Academic Editor: Vaibhav A. Diwadkar

Copyright (C 2011 Brian Dean. This is an open access article distributed under the Creative Commons Attribution License, which permits unrestricted use, distribution, and reproduction in any medium, provided the original work is properly cited.

\begin{abstract}
The search for clinically useful biomarkers has been one of the holy grails of schizophrenia research. This paper will outline the evolving notion of biomarkers and then outline outcomes from a variety of biomarkers discovery strategies. In particular, the impact of high-throughput screening technologies on biomarker discovery will be highlighted and how new or improved technologies may allow the discovery of either diagnostic biomarkers for schizophrenia or biomarkers that will be useful in determining appropriate treatments for people with the disorder. History tells those involved in biomarker research that the discovery and validation of useful biomarkers is a long process and current progress must always be viewed in that light. However, the approval of the first biomarker screen with some value in predicting responsiveness to antipsychotic drugs suggests that biomarkers can be identified and that these biomarkers that will be useful in diagnosing and treating people with schizophrenia.
\end{abstract}

\section{Introduction}

There is a growing recognition that the development of drugs with an increasing efficacy for reducing the psychotic symptoms of schizophrenia $(\mathrm{Sz})$ is slowing and there has been little progress in developing treatments for the negative symptoms and cognitive deficits associated with the disorder [1]. In addition, it is now acknowledged that the current symptom-based diagnoses of $\mathrm{Sz}$ defines a syndrome of disorders which are likely to have diverse pathophysiologies [2]. This hypothesis is supported by the existence of treatment resistant $\mathrm{Sz}$ [3] which does not appear to be primarily associated with the dopaminergic systems of the human CNS because they are the primary targets of antipsychotic drugs [4]. The limitation of having to study the syndrome of Sz is now recognised as hampering progress toward a better understanding of the pathophysiologies of its component disorders [2] whilst having to provide treatments at the level of the syndrome is a major factor preventing the development of personalised medicine [5]. As with other forms of medicine, the solution to both of these problems would be to divide subjects with $\mathrm{Sz}$ into more biologically homogenous subgroups using well validated biomarkers as diagnostic tests [6] which in some cases may result in separating groups into endophenotypes [7]. To fulfil the requirement of an endophenotype, subjects clustered by the use of a specific biomarker must have a disorder which is heritable, be primarily state independent and, within families, the endophenotype and illness cosegregate [8]. The differences between dissecting a syndrome using a diagnostic test or into endophenotypes is discussed extensively elsewhere [9]. Briefly, both a biomarker that can be used as a diagnostic test and the characteristics or tests used to define an endophenotype must be detectably different in the large majority, if not all, the subjects within the category defined by the biomarker or an endophenotype.

This paper will focus on the evolving notion of biomarkers and on the progress toward developing biomarkers that will allow subjects with $\mathrm{Sz}$ to be divided into more homogeneous subpopulations as a foundation to better understanding their pathophysiologies and responsiveness to treatments.

\section{The Evolving Concept of a Biomarker}

The initial definition of a biomarker was not focussed on purely medical requirements but defined a measurable event occurring in a biological system that could inform on the 
The Evolving notion of biomarkers



FIGURE 1: A schematic showing the evolving notion of biomarker utility from the inception of the concept of biomarkers to their use in medical research. This schema acknowledges that the discovery of diagnostic biomarkers is likely to also allow the separation of the differentcomponent disorders in the syndrome of schizophrenia and that more tailored treatment of these component disorder will be possible. Hence diagnostic biomarkers, to a greater or lesser extent, are also likely to be treatment biomarkers.

general state of an organism, its life expectancy, or the well being of its environment (Figure 1) [10]. These concepts saw biomarkers divided into three categories: exposure biomarkers, effect biomarkers, and susceptibility biomarkers [11]. An exposure biomarker might be a xenobiotic or metabolites that reflect contact with a harmful environmental factor (e.g., toxin), an effective biomarker was an endogenous component of an organism that would change after exposure to an environmental factor whilst a susceptibility marker would be an inherited factor that provided a measure of susceptibility or sensitivity to an environmental factor. It was proposed that the major advantages of identifying biomarkers for these three categories were to elucidate pathogenic mechanisms, to improve etiologic classification of environmentally related disease and to allow an early recognition of contact with disadvantageous environmental factors [12].

More recently, in the medical arena, biomarkers are viewed as measurable factors that can enable diagnoses [13], inform on disease pathophysiology [13], or enable decisions to be made on the optimal treatment of an individual (personalised medicine) [14]. The value of well-validated biomarkers now has strong face validity as drug regulatory bodies have, for example, acknowledged that certain biomarkers do have predictive validity for treatment responsiveness in some forms of cancers [14]. It is also well recognised that well-validated biomarkers can differentiate component disorders within diagnostic syndromes and that the separation of the component disorders within a syndrome leads to rapid advances in understanding the underlying pathophysiologies of those disorders $[13,15,16]$. Existing experience in biomarker validation has shown this process to be a far from trivial undertaking. For example, perhaps the earliest biomarker was the sweet taste of urine first used by Thomas Willis to identify subjects with diabetes in 1674 [17]. However, it was not until 1949 that Himsworth proposed two disorders within the syndrome that could be delineated based on the presence or absence of insulin resistance [18]. Over time, the form of diabetes that was not associated with insulin resistance became known as insulin-dependent diabetes mellitus and is now recognised as an autoimmune disease [19] that can be treated with immunotherapy [20]. By contrast, the form of diabetes associated with noninsulin-dependent diabetes mellitus is now primarily defined by the presence of insulin resistance [21] and initial treatments usually involve lifestyle changes and drugs that can lessen tissue insulin resistance [22]. Research is still ongoing to better understand and develop better treatments for both forms of diabetes.

Given lessons learned in other discipline, those in the psychiatry arena must acknowledge that biomarker discovery and validation will be difficult and time consuming. However, the rewards following the discovery of reliable biomarkers will be significant advances in understanding the causes and treatment of the component disorders within diagnostic syndrome such as Sz.

\section{Biomarker in Psychiatry}

The identification of biomarkers has been one of the holy grails for psychiatric research for many years [23]. Given that biomarkers are not widely used for either diagnoses or treatment monitoring of psychiatric disorders, the challenge of identifying clinically useful biomarkers must remain a high research priority. Recently, biomarker discovery has seen a significant boost with the availability of "omic" technologies that permit the large-scale rapid screening of DNA sequence (genome) [24], levels of gene expression (transcriptome) [25], levels of gene translation (proteome) [26], and levels of metabolic activity (metabolome) [27]. These technologies are now producing large data sets that could well be the foundation for identifying biomarkers 
that will be useful in diagnosis and directing treatment for serous psychiatric disorders such as Sz. Hence it would seem appropriate to review the progress that has been made towards identifying biomarkers that can be used to assist in the diagnoses and treatment of $\mathrm{Sz}$.

\section{Peripheral Biomarkers for Sz: Biochemical, Genetic, and Pharmacogenomic Approaches}

The initial search for biomarkers focussed on peripheral tissue. This was partly a pragmatic approach but also reflected the reality that a peripheral biomarker would have immediate clinical utility. One suggested diagnostic biomarker for $\mathrm{Sz}$ was the presence of 3,4-dimethoxyphenylethylamine in the urine of subjects with the disorder [28]. Importantly, when first reporting evidence to support this hypothesis, the authors stated that experiments would be required to validate the use of urinary 3,4-dimethoxyphenylethylamine as a biomarker for Sz. These subsequent experiments proved that urinary 3,4-dimethoxyphenylethylamine was not a biomarker for $\mathrm{Sz}$ and that there was doubt as to whether the "pink spot", by which the presence of 3,4dimethoxyphenylethylamine was measured, was a measure of that chemical entity [29]. Thus, the outcome from the initial experiments that suggested a diagnostic biomarker for $\mathrm{Sz}$ had been identified was disappointing but the ensuing debate led to the realisation that "perhaps the time has now finally come to stop investigating "schizophrenics" en masse, and to concentrate on individuals" [29]. Importantly, it is still acknowledged that the study of the syndrome of $\mathrm{Sz}$ is a major impediment to identifying the causes of the symptoms associated with the disorder [2]. It is also now clear that it is unlikely that a single biomarker will be identified for any particular symptom such as psychoses [30]. These two hypotheses acknowledge that $\mathrm{Sz}$ is a complex syndrome, however it was hoped that an extensive study of the human genome in subjects with $\mathrm{Sz}$ would reveal DNA sequence variation that were associated with an increased risk of developing the symptoms experience by people with the disorder [31] and hence lead to biomarkers with which to diagnose the disorder.

The notion that changes in DNA sequence could be associated with an increased susceptibility to Sz came from the recognition that the disorder was highly heritable [32]. The hypotheses that changes in the genome could be used to diagnose $\mathrm{Sz}$ gained significant support from the identification of susceptibility locus for $\mathrm{Sz}$ in chromosome 5 [33]. More extensive studies showed that there was not a susceptibility marker for $\mathrm{Sz}$ on chromosome 5 [34] but other studies have generated a large body of data that may lead to the identification of genetic biomarkers that could be used for either the diagnosis of $\mathrm{Sz}$ [35] or as an indicator of the best treatment for an individual with $\mathrm{Sz}$ with a particular genetic background (pharmacogenomics) [36]. Unfortunately, the failure of large-scale genomewide associations studies (GWAS) to identify strong genetic markers that can predict altered risk for $\mathrm{Sz}$ [37] suggests that no one genetic marker is associated with a major susceptibility risk for the disorder at the level of the syndrome. This has led to two proposals, one is that genetic associations with an altered risk for Sz can be discovered if larger cohorts are used to compensate for the search for low-effect size genetic associations [24] or that $\mathrm{Sz}$ does not result from genetic changes at the level of single nucleotide polymorphisms [37]. The failure to identify genetic markers that show an increased susceptibility for Sz may also support the argument that genetic testing may be more effective in identifying the risk of symptom severity level. This idea is supported by the reports of genetic markers associated with the severity of psychotic symptoms [38], cognitive deficits [39] and decline in IQ [40] in subjects with Sz. However, these findings need replication in large cohorts before they can be judged to be of clinical value.

Although many genetic studies have focussed on identifying diagnostic biomarker, other studies have used pharmacogenomics approaches to identify changes in DNA sequence that may provide indicators of treatment responsiveness based on genetic background [41]. Pharmacogenomic studies have shown that genetic polymorphisms in the cytochrome P450 gene can influence antipsychotic drug treatment responsiveness [41]. The cytochrome P450 gene is critical in antipsychotic drug metabolism and hence can be an indicator of antipsychotic drug clearance which is often inversely related to drug responsiveness [42]. It has also been suggested that polymorphism in the dopamine D2 receptor [43, 44], dopamine D3 receptors [43], the serotonin $2 \mathrm{~A}$ receptors [44], and catechol-O-methyl transferase [44] genes may be predictors of responsiveness to antipsychotic drugs and that polymorphisms in dopamine receptor genes and the serotonin $2 \mathrm{C}$ receptor gene may predict susceptibility for side-effects such as tardive dyskinesia and weight gain, respectively [43]. These findings have inherent face value as the proteins encoded by these genes are targeted by many antipsychotic drugs [45]. However, large-scale studies will be required to establish the clinical usefulness of these pharmacogenomics markers.

\section{Biomarker for Sz: Blood Based Studies}

Complimentary to studies using genetic material, predominantly obtained from white blood cells, there has been a long history of research trying to identify other blood-based markers for Sz. A lot of this research has focussed on the study of the human platelet which has some biochemical processes that are similar to those present in neurons [46, 47]. Many of the more recent studies have suggested that the functioning of the platelet may provide some indication as to likely antipsychotic drug responsiveness. For example, it has been suggested that low platelet serotonin 2A receptor levels prior to treatment may predict responsiveness to olanzapine [48] and that changes in dopamine uptake by platelets may provide a more generic indication of responsiveness to antipsychotic drug treatment [49]. Changes in basic platelet function, such as a blunted serotonin responsive aggregation [50] and neurotransmitter uptake [51], have also been suggested to be potential biomarkers for Sz. Finally, it has been reported that platelet functions such as dopamine uptake [52] and monoamine oxidase activity [53] may correlated with the severity symptoms such as delusions and auditory 
hallucinations, respectively. Unfortunately, many plateletbased findings need to be replicated in well-controlled studies involving large cohorts before their value as potential biomarkers can be fully assessed [54].

\section{Biomarker for Sz: High Throughput Screening of Blood and Brain Gene Expression}

Biomarker discovery research has been accelerated by the development of high-throughput screening techniques, such as microarrays, which allow levels of the expression of multiple genes in a tissue to be measured effectively in shorttime frame [55]. The initial study using such technology to probe the transcriptome in human postmortem CNS suggested that $\mathrm{Sz}$ was a disorder of dysregulated synapticrelated gene expression [56]. This finding was interesting given the hypothesis that $\mathrm{Sz}$ is a disorder that only occurs in humans [57] and that one of the major differences between humans and other species, at the level of gene expression, are changes in synaptic-related gene expression [58]. In addition, other studies examining changes in the transcriptome in the CNS of subjects with $\mathrm{Sz}$ suggest that changes in gene expression in Sz vary with duration of illness $[59,60]$. These findings raise the intriguing possibility that some biomarkers could be a measure of disease progression and may be useful in predicting changes in drug responsiveness that are known to occur with the progression of the disorder [61].

High-throughput screening presents the opportunity to bring together different findings relating to the pathophysiology of Sz that may prove seminal to biomarker discovery. Thus, microarray gene-expression studies using postmortem CNS tissue added to genetic studies using peripheral DNA (association studies) suggest a role of regulator of $\mathrm{G}$ protein signaling 4 in the pathophysiology of Sz [62]. This is an important finding in its own right but also serves to illustrate how a holistic approach using multiple technologies can serve to identify potential biomarkers for the disorder. In addition, combining results from studies using CNS and peripheral tissue is beginning to suggest that changes in CNS gene expression may, at least in part, be detected using peripheral tissue such as white blood cells. This hypothesis is supported by studies showing similar changes in gene expression levels for some genes in blood and brain tissue (e.g., Bcell translocation gene 1 , glycogen synthase kinase $3 \alpha$, major histocompatibility complex, class II, DR $\beta 1$, heterogeneous nuclear ribonucleoprotein A3, selenium binding protein 1, and splicing factor, arginine/serine-rich 1 [63]) from subjects with Sz. These findings are particularly important as they indicate it is possible to translate biomarkers identified using postmortem CNS into clinically useful tools because they can be measured in peripheral tissue and that, conversely, some changes in peripheral gene expression may provide insight into changes in brain gene expression in the CNS of subjects with Sz. Such conclusions must still be tempered by acknowledging other findings from microarray studies suggesting that changes in gene expression in peripheral blood cells are not present in the CNS from subjects with $\mathrm{Sz}$ $[64,65]$.
There are now intriguing data that could support the use of peripheral changes in gene expression as biomarkers for Sz. For example, changes in levels of selenium binding protein 1 expression have been found in peripheral tissue [63] and in the CNS from subjects with Sz [66]. However, the study of selenium-binding protein 1 expression in blood has shown that levels of gene expression correlate with levels of psychotic symptoms independent of whether the blood donor had $\mathrm{Sz}$ of bipolar disorder (BD) [63]. These latter data suggest that changes in gene expression may be associated with symptom severity and be associated with phenotypes rather than diagnoses [67]. If that is the case, gene expression biomarkers may be confirming the suggestion that the time has finally come to stop investigating schizophrenia in isolation as suggested over four decades ago [29]. Rather, it may be time for a more holistic approach testing potential biomarkers on a gene by gene basis carefully assessing their diagnostic potential but also whether they are pointing to commonalities in genetic susceptibility to multiple psychiatric disorders.

\section{Biomarker for Sz: High Throughput Screening of the Blood and Brain Proteome}

Whilst changes in levels of gene transcription at the level of mRNA may be a biomarker for $\mathrm{Sz}$ it is now recognised that not all changes in the transcriptome translate into changes in levels of the encoded protein [59] and hence the physiological significance of altered gene transcription is not readily apparent. Given one goal of biomarker discovery is to improve knowledge of disease pathophysiology, highthroughput methodologies that allow the study of the human blood and brain proteome have been used to identify potential biomarkers for Sz. This approach has been validated by a recent report that $25 \%$ of subjects with $\mathrm{Sz}$ can be separated into a separate population based on the loss of the majority $(\sim 75 \%)$ of their cortical muscarinic M1 receptors [68] which shows that levels of proteins can be used to identify subgroups of subjects within the syndrome of Sz. Moreover, the subjects with $\mathrm{Sz}$ with a marked loss in muscarinic M1 receptors have been shown to have altered muscarinic M1 receptor signalling [69] and increased levels of a microRNA that targets muscarinic M1 receptor mRNA to reduce protein translation [70] that are not present in people with $\mathrm{Sz}$ that do not have changes in muscarinic M1 receptors. These data support the hypothesis that (i) it should be possible to discover biomarkers for the identification of subgroups of subjects within the syndrome of $\mathrm{Sz}$ and (ii) once these subgroups of subjects have been identified it should be possible to understand their specific pathophysiologies. The findings on cortical muscarinic M1 receptors also argues against the position that the use of high-throughput technologies are essential for biomarker discovery in $\mathrm{Sz}$ [71].

A number of studies of the human proteome have claimed to identify biomarkers for Sz. One study using surface-enhanced laser desorption/ionization time of flight mass spectrometry and postmortem brain tissue suggests that there are at least 21 potential biomarkers (Table 1) 
TABLE 1: Proteins suggested as potential biomarkers for Sz or BPD due to diagnostic-specific changes detected using surface-enhanced laser desorption/ionization time of flight mass spectrometry in postmortem CNS.

\begin{tabular}{|c|c|}
\hline Diagnoses & Proposed biomarker \\
\hline $\mathrm{Sz}$ & $\begin{array}{l}\text { CD58 molecule } \\
\text { Calmodulin } 1 \text { (phosphorylase kinase, delta) } \\
\text { Mago-nashi homolog, proliferation-associated (Drosophila) } \\
\text { MYC associated factor X } \\
\text { Regulator of G-protein signaling } 11 \\
\text { Ubiquitin carboxyl-terminal hydroxylase isozyme L1 } \\
\text { Cyclophilin A } \\
\text { CEBPZ CCAAT/enhancer binding protein (C/EBP), zeta } \\
\text { LOC153154 similar to short heat shock protein } 60 \text { Hsp60s2 } \\
\text { Phosphoglycerate mutase } 1 \\
\text { Tyrosine 3-monooxygenase/tryptophan 5-monooxygenase activation protein, eta polypeptide } \\
\text { NK2 homeobox 4,2,4-dienoyl CoA reductase } 2 \\
\text { Peroxisomal, methylthioadenosine phosphorylase } \\
\text { Purine nucleoside phosphorylase } \\
\text { Bystin-like } \\
\text { Ankyrin repeat domain } 12 \\
\text { Proteasome (prosome, macropain) subunit, alpha type, } 7 \\
\text { Aldolase C, fructose-bisphosphate } \\
\text { Sphingomyelin phosphodiesterase } 1 \text {, acid lysosomal } \\
\text { Glutamate-ammonia ligase and enolase } 2 \text { (gamma, neuronal) }\end{array}$ \\
\hline BPD & $\begin{array}{l}\text { Myelin basic protein } \\
\text { Dickkopf homolog } 2 \text { (Xenopus laevis) } \\
\text { CEBPZ CCAAT/enhancer binding protein (C/EBP), zeta; 2,4-dienoyl CoA reductase 2, peroxisomal } \\
\text { Bystin-like } \\
\text { Ankyrin repeat domain } 12 \\
\text { Aldolase C, fructose-bisphosphate }\end{array}$ \\
\hline
\end{tabular}

that can be used to separate $\mathrm{Sz}$ and/or BD from controls [72]. Again these data suggest that not all biomarkers will always show diagnostic specificity but some may be useful in assessing common factors that cross psychiatric diagnostic boundaries. A study using postmortem brain tissue and the same technology to measure protein levels found that no single protein could be used as a diagnostic-specific biomarker for $\mathrm{Sz}$ [73] but that a cluster analysis of the intensities of several proteins were required to separate subjects with Sz with an accuracy of $70 \%$. Thus, whilst initial outcomes from the use of surface-enhanced laser desorption/ionization time of flight mass spectrometry are encouraging, there is now a need for follow-up data to support the proposed proteins, or groups of proteins, as biomarkers for Sz; of course these studies should also advance understanding of the pathophysiology of the disorder.

Using a liquid chromatography/mass spectrometry approach 10 proteins (CD5 molecule-like (CD5L), IGHM immunoglobulin heavy constant $\mathrm{mu}(\mathrm{IGHM})$, coagulation factor XIII, B polypeptide (F13B), transferring (TF), apolipoprotein D (APOD), apolipoprotein A-1 (APOA1), apolipoprotein A-IV (APOA4), apolipoprotein A-II (APOA2), apolipoprotein C-I (APOC1), and alpha-2-HS-glycoprotein (AHSG)) have been identified as altered in serum from drug naïve subjects with $\mathrm{Sz}$ compared to controls [74]. Clearly the identification of changes in the blood proteome in drug naive subjects is significant as the effects of antipsychotic drug treatment would not be a confound. Significantly, APOD
[75, 76], APOA1 [77-79], and TF [80, 81] have previously been identified as being altered in blood from subjects with Sz. In addition, APOA4 has been reported as altered in the CSF from subjects with $\mathrm{Sz}$ [82]. These findings obtained in protein-specific studies add to the hypotheses that blood proteins can be used as biomarkers in Sz.

Another recent approach to identify biomarkers for psychiatric disorders is a large study comparing proteins levels in plasma from subjects with $\mathrm{Sz}$, major depressive disorders (MDD), and control subjects [83]. In this study biomarker identification relied on a technology that examined levels of multiple chemokines, cytokines, hormones, growth factors, and antigens. Significantly, the study showed a greater separation of samples from subjects with $\mathrm{Sz}$ from controls than was achieved for MDD and controls using cluster analysis. The proteins that most strongly allowed the separation of $\mathrm{Sz}$ from controls and MDD were chemokine (C-C motif) ligand 4 (CCL4), chemokine (C-C motif) ligand 5 (CCL5), chemokine (C-C motif) ligand 22 (CCL22), chemokine (C-X-C motif) ligand 5 (CXCL5), TIMP metallopeptidase inhibitor 1 (TIMP1), epidermal growth factor (EGF), serpin peptidase inhibitor, clade A (alpha-1 antiproteinase, antitrypsin), member 7 (SERPINA7), and KIT ligand (KITLG). Conversely, changes in blood levels of insulin (INS), alpha-2-macroglobulin (A2M), matrix metallopeptidase 9 (gelatinase B, $92 \mathrm{kDa}$ gelatinase, and $92 \mathrm{kDa}$ type IV collagenase) (MMP3) and tumour necrosis factor receptor superfamily, member 1B (TNFRSF1B) allowed the 
separation of MDD from controls and subjects with Sz. Finally, brain-derived neurotrophic factor (BDNF), serpin peptidase inhibitor, clade $\mathrm{E}$ (nexin, plasminogen activator inhibitor type 1), member 1 (SERPINA1), and APODA1 allowed the separation of $\mathrm{Sz}$ and MDD from controls but did not show diagnostic specificity.

Reviewing results from blood-based biomarker studies critically, it is notable that there is no agreement on potential biomarkers across two studies. This could be due to many uncontrolled variables across the studies as blood proteins levels are known to vary with multiple factors including time of day [84], menstrual cycle [85], and food intake status [86]. Hence, at some point those involved in blood biomarker discovery for $\mathrm{Sz}$ should attempt to come to a consensus with regard to standardisation blood collecting with regards to fasting status, time of bleed, and other critical factors which can add variability to blood protein levels, as has been advocated for biomarker discovery for other diseases [87].

Another approach to identifying potential biomarkers at the level of the protein has been to study the CNS proteome in subjects with psychiatric disorders [88]. Whilst the study of the human CNS proteome in $\mathrm{Sz}$ has been ongoing for over a decade $[89,90]$ only 16 gray matter proteins (aldolase C, fructose-bisulphate, creatine kinase (brain), dynamin 1 , dihydropyrimidinase-like 2 , enolase $1 \alpha$, enolase $2(\gamma$ neuronal), glial fibrillary acidic protein, glutamate-ammonia ligase, guanine nucleotide binding protein $\beta$ polypeptide 1 , internexin neuronal intermediate filament protein $\alpha$, and $N$ ethylmaleimide-sensitive factor) and 8 white matter proteins (dihydropyrimidinase-like 2, inositol-monophosphatase 1, neurofilament, light polypeptide, Parkinson Disease 7, 14-33 zeta, 1-lactate dehydrogenase Chain B, and stathmin 1) have been reported as altered in the same direction in more than one study of the human proteome [91]. Of equal concern is that some of these proteins, such as dynamin 1 [92], $N$-ethylmaleimide-sensitive factor [93], glial fibrillary acidic protein [94], and 14-3-3 zeta [95], have been reported to be unaltered in the CNS of subjects with Sz when measured using Western blotting. However, it has to be acknowledged that current studies on the human CNS proteome have been completed using relatively small number of cases and this is an issue given the need for the adequately powered cohort sizes needed when attempting to identify clinically valid biomarkers in any form of disease [96].

\section{Towards Clinically Useful Biomarkers for $\mathrm{Sz}$}

There is a high expectation that the discovery of biomarkers will greatly accelerate our understanding of the pathophysiology of syndromes such as $\mathrm{Sz}$ [2]. This high expectation brings the danger that "apparent" failures to identify useful biomarkers may lead to the perception that biomarker discovery is an impossible goal. However, biomarker discovery in $\mathrm{Sz}$ is still in its infancy and the likelihood of identifying biomarkers is dependent on a number of factors, not the least is the ongoing development of the high-throughput technologies that allow the probing of the human genome, transcriptome, proteome, and metabolome.



FIGURE 2: The number of protein spots visualised in Brodmann's area 46 from subjects with $\mathrm{Sz}$, bipolar disorder, and schizophrenia after differential detergent solubilisation and separation across 5 two dimensional gels.

In this respect, deep sequencing is a new technology that will allow a much more comprehensive analysis of the human genome in much larger cohorts of subjects with disorders such as Sz [97] and this technology may be able to identify genetic biomarkers even in a complex syndrome such as $\mathrm{Sz}$ [31]. At the level of transcriptomics, an increasing knowledge of the complexities of gene expression is producing technologies that can probe mRNA at ever increasing levels of complexity [98]. In addition, there are efforts to refine existing technologies [99] and utilise new technologies [100, 101] to increase the proportion of the human proteome that can be effectively measured in blood, CNS, or other tissues.

The capacity to significantly increase the reach of proteomics technologies in psychiatric disease is illustrated by the application of a differential-detergent solubility, multigel approach to analysis of the human CNS proteome in tissue from subjects with $\mathrm{Sz}, \mathrm{BD}$, and control subjects [99]. This approach has led to the visualisation of approximately 3,800 protein spots in the acidic protein fraction from cortex and caudate-putamen, this is close to triple the number of protein spots visualised using crude homogenates as reported in most studies from subjects with $\mathrm{Sz}$ [99].

This methodology has now been applied to studying the acidic and basic proteins in Brodmann's area 46 (BA 46) from subjects with $\mathrm{Sz}, \mathrm{BD}$, and controls with 8 subjects in each cohort. Using this methodology, a total of 8152 protein spots were visualised across all of the cases studied, of which 5590 $(69 \%)$ were present in two or more cases. Significantly, as is the case in some studies, if data analyses was limited to the study of protein spots visualised in every case then only 245 protein spots $(3 \%)$ would be included for analyses. This would exclude the analyses of spots that seemed to have a significant variation with diagnoses (e.g., present in $>75 \%$ of controls and $<25 \%$ of cases with $\mathrm{Sz}$ ). At the level of disease cohorts, the highest number of protein spots were visualised in tissue from subjects with $\mathrm{Sz}(n=5917)$, followed by tissue 


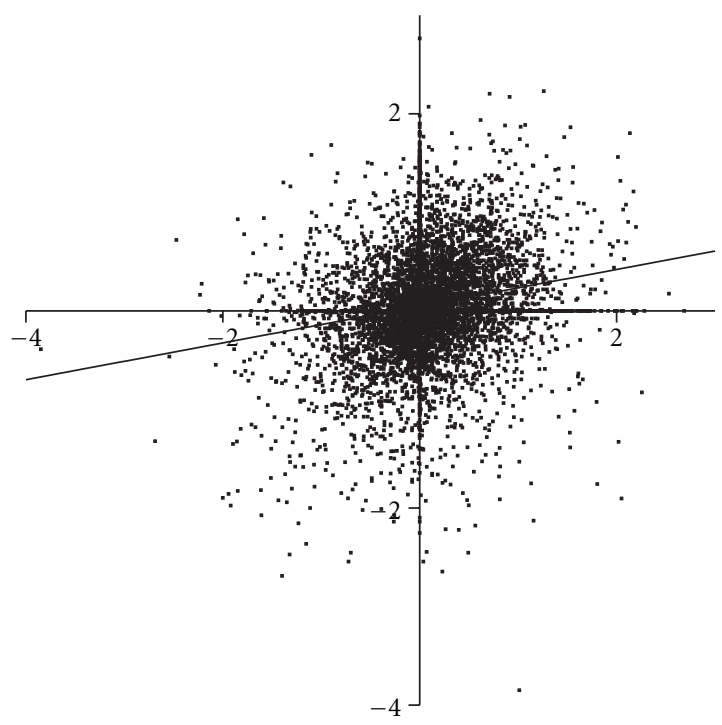

FIGURE 3: A comparison of the intensity of protein spots, normalised to the intensity of the corresponding protein spot in control CNS, in Brodmann's area 46 from subjects with Sz (Y-axis) and bipolar disorder (X-axis). Spots in the upper left quadrant show increased levels in Sz compared to bipolar disorder whereas those in the lower right quadrant show increased levels of proteins in bipolar disorder with lower levels in Sz.

from subjects with $\mathrm{BD}(n=5746)$ and the fewest spots were detected in tissue from control subjects $(n=4946)$. These data are interesting as they suggest that subjects with $\mathrm{Sz}$ and $\mathrm{BD}$ expressed more CNS proteins or have overall higher levels of lower abundance CNS proteins, than do control subjects. Importantly, 3503 (59\%) protein spots were detected in two or more subjects with $\mathrm{Sz}, 3322(58 \%)$ in two or more subjects with $\mathrm{BD}$ and $3211(65 \%)$ in two or more of the control subjects (Figure 2).

The data from a simple modification of an established methodology serve to show how existing technologies can still have value in the study of the human CNS proteome. The increased utility of such modifications can be illustrated by comparing the intensity of each protein spot, for protein present in at least two cases, from subjects with $\mathrm{Sz}$ and $\mathrm{BD}$ normalised to the intensity of the same spot in the proteome of control subjects (Figure 3). These data show that differential changes in many relative protein intensities are occurring in the CNS of subjects of Sz and BD and the challenge is to mine this rich data set to identify candidate proteins that may prove valuable as biomarkers to separate the two disorders.

\section{Conclusions}

There are clear and urgent needs to develop biomarkers to aid in the diagnoses and monitoring of treatment in subjects with psychiatric disorders such as Sz [5]. It will be important not to grow impatient and prematurely discard the hypothesis that biomarkers can be identified to aid in the diagnoses and treatment of the disorder. For example, in diabetes, the development of clinically useful biomarkers has spanned several centuries from the discovery of sweet urine to immunological diagnostic tests for certain forms of diabetes [13]. Moreover, the recognition of the Federal Drug Authority that at least one pharmacogenomics test (based on genetic variation in cytochrome P450) is of value in assessing likely treatment responsiveness in people with $\mathrm{Sz}$ [102] suggest that progress is being made toward biomarker discovery, validation and eventually widespread clinical use. This being said, those in the biomarker research space need to be considering some form of standardisation of blood collection to attempt to minimise blood protein level variation due to factors other than disease pathophysiology or treatment response. In addition, those involved in biomarker research using postmortem CNS or neuroimaging need to develop networks that will facilitate a rapid validation of potential biomarkers across brain collections and between imaging centres. Both these strategies may be essential for the rigorous testing of potential biomarkers to occur rapidly as a prelude to moving such validated biomarkers into the clinic.

\section{Acknowledgments}

The author would like to acknowledge Geoffrey Pavey, Simone Boer, and Alison Digney for assisting in running and analysing 2D gels to visualise proteins in Brodmann's area 46 from subjects with schizophrenia, bipolar disorder, and controls. The work described in the manuscript was supported in part by NHMRC Grant no. APP1002240, NHMRC Grant no. 192339, R01 MH069696-01 and grants-in aid from the Rebecca L. Cooper Medical Research Foundation. The study was supported by Operational Infrastructure Support (OIS) from the Victorian State Government.

\section{References}

[1] T. R. Insel, "Disruptive insights in psychiatry: transforming a clinical discipline," Journal of Clinical Investigation, vol. 119, no. 4, pp. 700-705, 2009.

[2] C. A. Tamminga, "Accelerating new knowledge in schizophrenia," American Journal of Psychiatry, vol. 165, no. 8, pp. 949-951, 2008.

[3] H. Elkis, "Treatment-Resistant Schizophrenia," Psychiatric Clinics of North America, vol. 30, no. 3, pp. 511-533, 2007.

[4] S. Miyamoto, G. E. Duncan, C. E. Marx, and J. A. Lieberman, "Treatments for schizophrenia: a critical review of pharmacology and mechanisms of action of antipsychotic drugs," Molecular Psychiatry, vol. 10, no. 1, pp. 79-104, 2005.

[5] L. J. Lesko, "Personalized medicine: elusive dream or imminent reality?" Clinical Pharmacology and Therapeutics, vol. 81, no. 6, pp. 807-816, 2007.

[6] E. Schwarz and S. Bahn, "The utility of biomarker discovery approaches for the detection of disease mechanisms in psychiatric disorders," British Journal of Pharmacology, vol. 153, supplement 1, pp. S133-S136, 2008.

[7] T. R. Insel and B. N. Cuthbert, "Endophenotypes: bridging Genomic Complexity and Disorder Heterogeneity," Biological Psychiatry, vol. 66, no. 11, pp. 988-989, 2009.

[8] I. I. Gottesman and T. D. Gould, "The endophenotype concept in psychiatry: etymology and strategic intentions," 
American Journal of Psychiatry, vol. 160, no. 4, pp. 636-645, 2003.

[9] C. L. Arfken, S. Carney, and N. N. Boutros, "Translating biological parameters into clinically useful diagnostic tests," Current Psychiatry Reports, vol. 11, no. 4, pp. 320-323, 2009.

[10] Committee on Biological Markers of the National Research Council, "Biological markers in environmental health research," Environmental Health Perspectives, vol. 74, pp. 1191, 1987.

[11] P. Grandjean, "Biomarkers in epidemiology," Clinical Chemistry, vol. 41, no. 12, pp. 1800-1803, 1995.

[12] B. S. Hulka and T. Wilcosky, "Biological markers in epidemiologic research," Archives of Environmental Health, vol. 43, no. 2, pp. 83-89, 1988.

[13] E. A. M. Gale, "Perspectives in diabetes: the discovery of type 1 diabetes," Diabetes, vol. 50, no. 2, pp. 217-226, 2001.

[14] J. R. Johnson, G. Williams, and R. Pazdur, "End points and United States Food and Drug Administration approval of oncology drugs," Journal of Clinical Oncology, vol. 21, no. 7, pp. 1404-1411, 2003.

[15] S. M. Hewitt, J. Dear, and R. A. Star, "Discovery of protein biomarkers for renal diseases," Journal of the American Society of Nephrology, vol. 15, no. 7, pp. 1677-1689, 2004.

[16] D. W. Benson, "Advances in cardiovascular genetics and embryology: role of transcription factors in congenital heart disease," Current Opinion in Pediatrics, vol. 12, no. 5, pp. 497500, 2000.

[17] F. Henschen, "On the term diabetes in the works of Aretaeus and Galen," Medical History, vol. 13, no. 2, pp. 190-192, 1969.

[18] H. P. Himsworth, "The syndrome of diabetes mellitus and its causes," The Lancet, vol. 253, no. 6551, pp. 465-473, 1949.

[19] J. F. Bach, "Insulin-dependent diabetes mellitus as an autoimmune disease," Endocrine Reviews, vol. 15, no. 4, pp. 516-542, 1994.

[20] J. J. Cook, I. Hudson, L. C. Harrison et al., "Doubleblind controlled trial of azathioprine in children with newly diagnosed type I diabetes," Diabetes, vol. 38, no. 6, pp. 779$783,1989$.

[21] E. Ferrannini, "Insulin resistance versus insulin deficiency in non-insulin-dependent diabetes mellitus: problems and prospects," Endocrine Reviews, vol. 19, no. 4, pp. 477-490, 1998.

[22] A. J. Krentz and C. J. Bailey, "Oral antidiabetic agents: current role in type 2 diabetes mellitus," Drugs, vol. 65, no. 3, pp. 385411, 2005.

[23] R. Noll, "The blood of the insane," History of Psychiatry, vol. 17, no. 4, pp. 395-418, 2006.

[24] P. F. Sullivan, "The psychiatric GWAS consortium: big science comes to psychiatry," Neuron, vol. 68, pp. 182-186, 2010.

[25] M. T. Tsuang, N. Nossova, T. Yager et al., "Assessing the validity of blood-based gene expression profiles for the classification of schizophrenia and bipolar disorder: a preliminary report," American Journal of Medical Genetics: Part B, vol. 133, no. 1, pp. 1-5, 2005.

[26] M. J. Romeo, V. Espina, M. Lowenthal, B. H. Espina, E. F. Petricoin III, and L. A. Liotta, "CSF proteome: a protein repository for potential biomarker identification," Expert Review of Proteomics, vol. 2, no. 1, pp. 57-70, 2005.

[27] R. Kaddurah-Daouk and K. R. R. Krishnan, "Metabolomics: a global biochemical approach to the study of central nervous system diseases," Neuropsychopharmacology, vol. 34, no. 1, pp. 173-186, 2009.
[28] A. J. Friedhoff and E. V. Winkle, "Isolation and characterization of a compound from the urine of schizophrenics," Nature, vol. 194, no. 4831, pp. 897-898, 1962.

[29] Editorial, "Lessons of the "Pink Spot"," British Medical Journal, vol. 18, p. 383, 1967.

[30] R. Freedman, R. Ross, S. Leonard et al., "Early biomarkers of psychosis," Dialogues in Clinical Neuroscience, vol. 7, no. 1, pp. 17-29, 2005.

[31] A. K. Tiwari, C. C. Zai, D. J. Muller, and Kennedy J. L., "Genetics in schizophrenia: where are we and what next?" Dialogues in Clinical Neuroscience, vol. 12, pp. 289-303, 2010.

[32] A. R. Jensen, "Estimation of the limits of heritability of traits by comparison of monozygotic and dizygotic twins," Proceedings of the National Academy of Sciences of the United States of America, vol. 58, no. 1, pp. 149-156, 1967.

[33] R. Sherrington, J. Brynjolfsson, H. Petursson et al., "Localization of a susceptibility locus for schizophrenia on chromosome 5," Nature, vol. 336, no. 6195, pp. 164-167, 1988.

[34] S. D. Detera-Wadleigh, L. R. Goldin, R. Sherrington et al., "Exclusion of linkage to 5q11-13 in families with schizophrenia and other psychiatric disorders," Nature, vol. 340, no. 6232, pp. 391-393, 1989.

[35] M. Gill, G. Donohoe, and A. Corvin, "What have the genomics ever done for the psychoses?" Psychological Medicine, vol. 40, no. 4, pp. 529-540, 2010.

[36] O. Blanc, G. Brousse, A. Meary, M. Leboyer, and P. M. Llorca, "Pharmacogenetic of response efficacy to antipsychotics in schizophrenia: pharmacodynamic aspects. Review and implications for clinical research," Fundamental and Clinical Pharmacology, vol. 24, no. 2, pp. 139-160, 2010.

[37] A. S. Bassett, S. W. Scherer, and L. M. Brzustowicz, "Copy number variations in schizophrenia: critical review and new perspectives on concepts of genetics and disease," American Journal of Psychiatry, vol. 167, no. 8, pp. 899-914, 2010.

[38] A. K. Malhotra, A. Breier, D. Goldman, L. Picken, and D. Pickar, "The apolipoprotein E $\varepsilon 4$ allele is associated with blunting of ketamine-Induced psychosis in schizophrenia: a preliminary report," Neuropsychopharmacology, vol. 19, no. 5, pp. 445-448, 1998.

[39] D. L. Liao, C. J. Hong, H. M. Chen et al., "Association of muscarinic M1 receptor genetic polymorphisms with psychiatric symptoms and cognitive function in schizophrenic patients," Neuropsychobiology, vol. 48, no. 2, pp. 72-76, 2003.

[40] K. E. Burdick, T. E. Goldberg, B. Funke et al., "DTNBP1 genotype influences cognitive decline in schizophrenia," Schizophrenia Research, vol. 89, no. 1-3, pp. 169-172, 2007.

[41] A. Mihaljević-Peleš, M. Šagud, N. Božina, M. Živković, and N. Jovanović, "Pharmacogenetics and antipsychotics in the light of personalized pharmacotherapy," Psychiatria Danubina, vol. 22, no. 2, pp. 335-337, 2010.

[42] N. Fleeman, Y. Dundar, R. Dickson et al., "Cytochrome P450 testing for prescribing antipsychotics in adults with schizophrenia: systematic review and meta-analyses," Pharmacogenomics Journal, vol. 11, no. 1, pp. 1-14, 2011.

[43] M. J. Arranz and J. De Leon, "Pharmacogenetics and pharmacogenomics of schizophrenia: a review of last decade of research," Molecular Psychiatry, vol. 12, no. 8, pp. 707-747, 2007.

[44] Y. Yamanouchi, N. Iwata, T. Suzuki, T. Kitajima, M. Ikeda, and N. Ozaki, "Effect of DRD2, 5-HT2A, and COMT genes on antipsychotic response to risperidone," Pharmacogenomics Journal, vol. 3, no. 6, pp. 356-361, 2003. 
[45] F. P. Bymaster, S. K. Hemrick-Luecke, K. W. Perry, and R. W. Fuller, "Neurochemical evidence for antagonism by olanzapine of dopamine, serotonin, $\alpha$-adrenergic and muscarinic receptors in vivo in rats," Psychopharmacology, vol. 124, no. 1-2, pp. 87-94, 1996.

[46] S. M. Stahl and H. Y. Meltzer, "The human platelet as a model for the dopaminergic neuron: kinetic and pharmacologic properties and the role of amine storage granules," Experimental Neurology, vol. 59, no. 1, pp. 1-15, 1978.

[47] W. B. Abrams and H. M. Solomon, "The human platelet as a pharmacologic model for the adrenergic neuron. The uptake and release of norepinephrine," Clinical Pharmacology and Therapeutics, vol. 10, no. 5, pp. 702-709, 1969.

[48] B. Arranz, P. Rosel, L. San et al., "Low baseline serotonin$2 \mathrm{~A}$ receptors predict clinical response to olanzapine in firstepisode schizophrenia patients," Psychiatry Research, vol. 153, no. 2, pp. 103-109, 2007.

[49] S. Sundram, B. Dean, and D. L. Copolov, "Typical and atypical neuroleptic drugs decrease platelet H-dopamine uptake in the rat," Psychiatry Research, vol. 62, no. 3, pp. 259263, 1996.

[50] R. Reddy, M. Keshavan, and J. K. Yao, "Blunted serotonergic responsivity in neuroleptic-naïve patients at first-episode of schizophrenia," Schizophrenia Research, vol. 90, no. 1-3, pp. 81-85, 2007.

[51] M. Zucker, A. Valevski, A. Weizman, and M. Rehavi, "Increased platelet vesicular monoamine transporter density in adult schizophrenia patients," European Neuropsychopharmacology, vol. 12, no. 4, pp. 343-347, 2002.

[52] B. Dean, J. Kulkarni, D. L. Copolov, P. Shrikanthan, V. Malone, and C. Hill, "Dopamine uptake by platelets from subjects with schizophrenia: a correlation with the delusional state of the patient," Psychiatry Research, vol. 41, no. 1, pp. 17-24, 1992

[53] H. Y. Meltzer and J. L. Zureick, "Relationship of auditory hallucinations and paranoia to platelet MAO activity in schizophrenics: sex and race interactions," Psychiatry Research, vol. 22, no. 2, pp. 99-109, 1987.

[54] P. Polister, "Reliability, decision rules, and the value of repeated tests," Medical Decision Making, vol. 2, no. 1, pp. 4769, 1982.

[55] A. T. Weeraratna, J. E. Nagel, V. De Mello-Coelho, and D. D. Taub, "Gene expression profiling: from microarrays to medicine," Journal of Clinical Immunology, vol. 24, no. 3, pp. 213-224, 2004.

[56] K. Mirnics and J. Pevsner, "Progress in the use of microarray technology to study the neurobiology of disease," Nature Neuroscience, vol. 7, no. 5, pp. 434-439, 2004.

[57] T. J. Crow, "Is schizophrenia the price that Homo sapiens pays for language?" Schizophrenia Research, vol. 28, no. 2-3, pp. 127-141, 1997.

[58] B. Dean, "Is schizophrenia the price of human central nervous system complexity?" Australian and New Zealand Journal of Psychiatry, vol. 43, no. 1, pp. 13-24, 2009.

[59] B. Dean, D. Keriakous, E. Scarr, and E. A. Thomas, "Gene expression profiling in Brodmann's area 46 from subjetcs with schizophrenia," Australian and New Zealand Journal of Psychiatry, vol. 41, no. 4, pp. 308-320, 2007.

[60] S. Narayan, B. Tang, S. R. Head et al., "Molecular profiles of schizophrenia in the CNS at different stages of illness," Brain Research, vol. 1239, no. C, pp. 235-248, 2008.

[61] M. E. Kelley, G. L. Haas, and D. P. van Kammen, "Longitudinal progression of negative symptoms in schizophrenia: a new look at an old problem," Schizophrenia Research, vol. 105, no. 1-3, pp. 188-196, 2008.

[62] K. Mirnics, F. A. Middleton, G. D. Stanwood, D. A. Lewis, and P. Levitt, "Disease-specific changes in regulator of G-protein signaling 4 (RGS4) expression in schizophrenia," Molecular Psychiatry, vol. 6, no. 3, pp. 293-301, 2001.

[63] S. J. Glatt, I. P. Everall, W. S. Kremen et al., "Comparative gene expression analysis of blood and brain provides concurrent validation of SELENBP1 up-regulation in schizophrenia," Proceedings of the National Academy of Sciences of the United States of America, vol. 102, no. 43, pp. 15533-15538, 2005.

[64] N. A. Bowden, J. Weidenhofer, R. J. Scott et al., "Preliminary investigation of gene expression profiles in peripheral blood lymphocytes in schizophrenia," Schizophrenia Research, vol. 82, no. 2-3, pp. 175-183, 2006.

[65] M. Takahashi, H. Hayashi, Y. Watanabe et al., "Diagnostic classification of schizophrenia by neural network analysis of blood-based gene expression signatures," Schizophrenia Research, vol. 119, no. 1-3, pp. 210-218, 2010.

[66] T. Kanazawa, G. Chana, S. J. Glatt et al., "The utility of selenbp1 gene expression as a biomarker for major psychotic disorders: replication in schizophrenia and extension to bipolar disorder with psychosis," American Journal of Medical Genetics, Part B, vol. 147, no. 6, pp. 686-689, 2008.

[67] A. B. Niculescu, L. L. Lulow, C. A. Ogden et al., "PhenoChipping of psychotic disorders: a novel approach for deconstructing and quantitating psychiatric phenotypes," American Journal of Medical Genetics, Part B, vol. 141, no. 6, pp. 653-662, 2006.

[68] E. Scarr, T. F. Cowie, S. Kanellakis, S. Sundram, C. Pantelis, and B. Dean, "Decreased cortical muscarinic receptors define a subgroup of subjects with schizophrenia," Molecular Psychiatry, vol. 14, no. 11, pp. 1017-1023, 2009.

[69] H. Salah-Uddin, E. Scarr, G. Pavey et al., "Altered M Muscarinic Acetylcholine Receptor (CHRM1)-G $\alpha$ Coupling in a Schizophrenia Endophenotype," Neuropsychopharmacology, vol. 34, no. 9, pp. 2156-2166, 2009.

[70] M. J. Cairns, N. Beveridge, E. Scarr, and B. Dean, "A potential role for gene directed microRNAs in the pathophysiology of muscarinic receptor deficit schizophrenia," Australian and New Zealand Journal of Psychiatry, vol. 44, supplement 1, p. A10, 2010.

[71] E. Schwarz and S. Bahn, "Biomarker discovery in psychiatric disorders," Electrophoresis, vol. 29, no. 13, pp. 2884-2890, 2008.

[72] S. I. Novikova, F. He, N. J. Cutrufello, and M. S. Lidow, "Identification of protein biomarkers for schizophrenia and bipolar disorder in the postmortem prefrontal cortex using SELDI-TOF-MS ProteinChip profiling combined with MALDI-TOF-PSD-MS analysis," Neurobiology of Disease, vol. 23, no. 1, pp. 61-76, 2006.

[73] J. Mei, D. Kolbin, H. T. Kao, and B. Porton, "Protein expression profiling of postmortem brain in schizophrenia," Schizophrenia Research, vol. 84, no. 2-3, pp. 204-213, 2006.

[74] Y. Levin, L. Wang, E. Schwarz, D. Koethe, F. M. Leweke, and S. Bahn, "Global proteomic profiling reveals altered proteomic signature in schizophrenia serum," Molecular Psychiatry, 2009.

[75] E. A. Thomas, B. Dean, G. Pavey, and J. G. Sutcliffe, "Increased CNS levels of apolipoprotein D in schizophrenic and bipolar subjects: implications for the pathophysiology of psychiatric disorders," Proceedings of the National Academy of Sciences of the United States of America, vol. 98, no. 7, pp. 4066-4071, 2001. 
[76] S. P. Mahadik, M. M. Khan, D. R. Evans, and V. V. Parikh, "Elevated plasma level of apolipoprotein D in schizophrenia and its treatment and outcome," Schizophrenia Research, vol. 58, no. 1, pp. 55-62, 2002.

[77] J. T. J. Huang, L. Wang, S. Prabakaran et al., "Independent protein-profiling studies show a decrease in apolipoprotein A1 levels in schizophrenia CSF, brain and peripheral tissues," Molecular Psychiatry, vol. 13, no. 12, pp. 1118-1128, 2008.

[78] Y. J. La, C. L. Wan, H. Zhu et al., "Decreased levels of apolipoprotein A-I in plasma of schizophrenic patients," Journal of Neural Transmission, vol. 114, no. 5, pp. 657-663, 2007.

[79] Y. Yang, C. Wan, H. Li et al., "Altered levels of acute phase proteins in the plasma of patients with schizophrenia," Analytical Chemistry, vol. 78, no. 11, pp. 3571-3576, 2006.

[80] C. T. Wong, W. F. Tsoi, and N. Saha, "Acute phase proteins in male Chinese schizophrenic patients in Singapore," Schizophrenia Research, vol. 22, no. 2, pp. 165-171, 1996.

[81] E. Bock, B. Weeke, and O. J. Rafaelsen, "Serum proteins in acutely psychotic patients," Journal of Psychiatric Research, vol. 9, no. 1, pp. 1-9, 1971.

[82] L. Jiang, K. Lindpaintner, H. F. Li et al., "Proteomic analysis of the cerebrospinal fluid of patients with schizophrenia," Amino Acids, vol. 25, no. 1, pp. 49-57, 2003.

[83] E. Domenici, D. R. Willé, F. Tozzi et al., "Plasma protein biomarkers for depression and schizophrenia by multi analyte profiling of case-control collections," PLOS ONE, vol. 5, no. 2, article e9166, 2010.

[84] E. Van Cauter, R. Leproult, and D. J. Kupfer, "Effects of gender and age on the levels and circadian rhythmicity of plasma cortisol," Journal of Clinical Endocrinology and Metabolism, vol. 81, no. 7, pp. 2468-2473, 1996.

[85] K. P. McNatty, D. T. Baird, and A. Bolton, "Concentration of oestrogens and androgens in human ovarian venous plasma and follicular fluid throughout the menstrual cycle," Journal of Endocrinology, vol. 71, no. 1, pp. 77-85, 1976.

[86] P. C. Butler, J. Chou, W. B. Carter et al., "Effects of meal ingestion on plasma amylin concentration in NIDDM and nondiabetic humans," Diabetes, vol. 39, no. 6, pp. 752-756, 1990.

[87] F. D. Sistare, F. Dieterle, S. Troth et al., "Towards consensus practices to qualify safety biomarkers for use in early drug development," Nature Biotechnology, vol. 28, no. 5, pp. 446$454,2010$.

[88] B. Dean, D. Keriakous, E. A. Thomas, and E. Scarr, "Understanding the pathology of schizophenia: the impact of high-throughput screening of the genome and proteome in postmortem CNS," Current Psychiatry Reviews, vol. 1, pp. $1-9,2005$.

[89] P. F. Edgar, S. J. Schonberger, B. Dean, R. L. M. Faull, R. Kydd, and G. J. S. Cooper, "A comparative proteome analysis of hippocampal tissue from schizophrenic and Alzheimer's disease individuals," Molecular Psychiatry, vol. 4, no. 2, pp. 173-178, 1999.

[90] P. F. Edgar, J. E. Douglas, G. J. S. Cooper, B. Dean, R. Kydd, and R. L. M. Faull, "Comparative proteome analysis of the hippocampus implicates chromosome 6q in schizophrenia," Molecular Psychiatry, vol. 5, no. 1, pp. 85-90, 2000.

[91] J. A. English, K. Pennington, M. J. Dunn, and D. R. Cotter, "The neuroproteomics of schizophrenia," Biological Psychiatry, vol. 69, no. 2, pp. 163-172, 2011.

[92] E. Scarr, L. Gray, D. Keriakous, P. J. Robinson, and B. Dean, "Increased levels of SNAP-25 and synaptophysin in the dorsolateral prefrontal cortex in bipolar I disorder," Bipolar Disorders, vol. 8, no. 2, pp. 133-143, 2006.

[93] L. Gray, E. Scarr, and B. Dean, "N-Ethylmaleimide sensitive factor in the cortex of subjects with schizophrenia and bipolar I disorder," Neuroscience Letters, vol. 391, no. 3, pp. 112-115, 2006.

[94] B. Dean, L. Gray, and E. Scarr, "Regionally specific changes in levels of cortical $S 100 \beta$ in bipolar 1 disorder but not schizophrenia," Australian and New Zealand Journal of Psychiatry, vol. 40, no. 3, pp. 217-224, 2006.

[95] B. Dean, S. A. Boer, A. Mackinnon, and M. Berk, "CNS 14-3-3 $\zeta$ : changes with sex but not psychiatric diagnoses or psychotropic drug treatment," Schizophrenia Research, vol. 93, no. 1-3, pp. 51-57, 2007.

[96] N. Rifai, M. A. Gillette, and S. A. Carr, "Protein biomarker discovery and validation: the long and uncertain path to clinical utility," Nature Biotechnology, vol. 24, no. 8, pp. 971983, 2006.

[97] I. Rudan, "New technologies provide insights into genetic basis of psychiatric disorders and explain their co-morbidity," Psychiatria Danubina, vol. 22, no. 2, pp. 190-192, 2010.

[98] D. D. Licatalosi and R. B. Darnell, "RNA processing and its regulation: global insights into biological networks," Nature Reviews Genetics, vol. 11, no. 1, pp. 75-87, 2010.

[99] B. Dean, G. Pavey, and A. I. Smith, "Using differential solubilization and 2-D gel electrophoresis to visualize increased numbers of proteins in the human cortex and caudate nucleus and putamen," Proteomics-Clinical Applications, vol. 2, no. 9, pp. 1281-1289, 2008.

[100] E. Cerasoli, P. D. Rakowska, A. Horgan et al., "MiSMALDI: microgel-selected detection of protein biomarkers by MALDI-ToF mass spectrometry," Molecular BioSystems, vol. 6, no. 11, pp. 2214-2217, 2010.

[101] M. Ndao, A. Rainczuk, M. C. Rioux, T. W. Spithill, and B. J. Ward, "Is SELDI-TOF a valid tool for diagnostic biomarkers?" Trends in parasitology, vol. 26, pp. 561-567, 2010.

[102] J. De Leon, S. C. Armstrong, and K. L. Cozza, "Clinical guidelines for psychiatrists for the use of pharmacogenetic testing for CYP450 2D6 and CYP450 2C19," Psychosomatics, vol. 47 , no. 1 , pp. $75-85,2006$. 




The Scientific World Journal


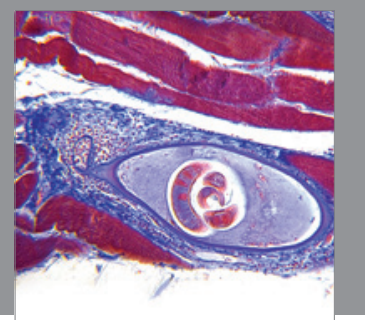

Gastroenterology

Research and Practice
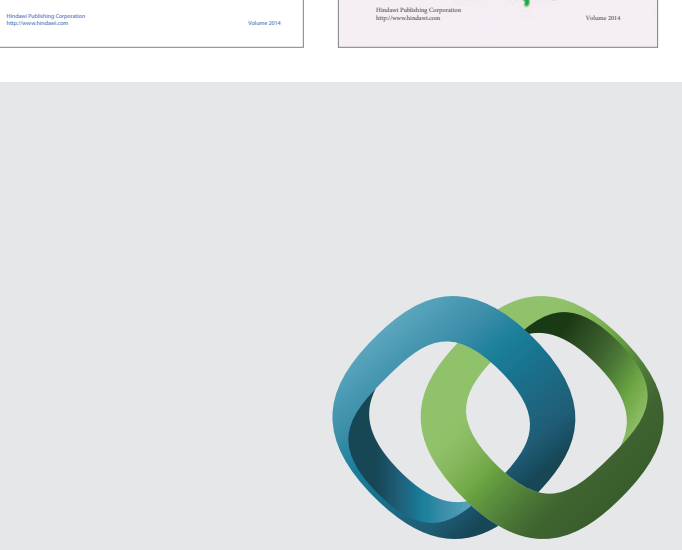

\section{Hindawi}

Submit your manuscripts at

http://www.hindawi.com
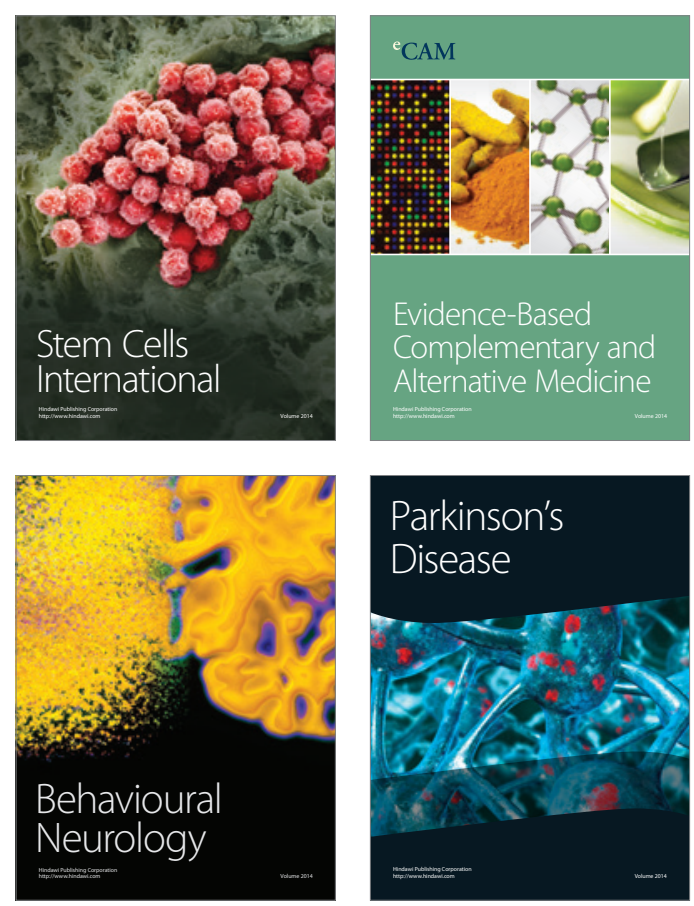

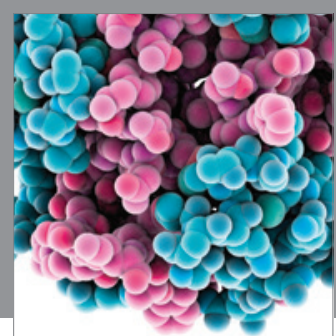

Journal of
Diabetes Research



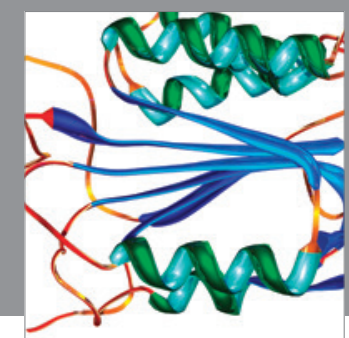

Disease Markers
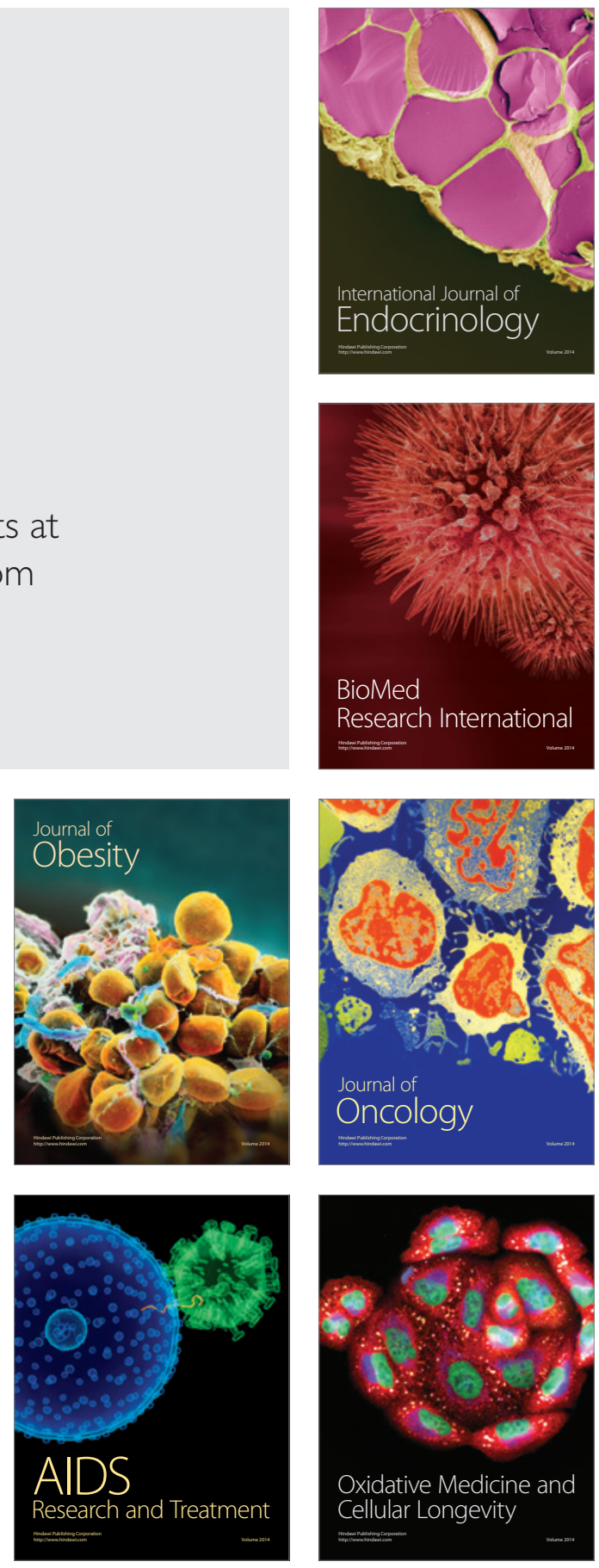